\section{Fragilitätsfrakturen: Osteoporose-Diagnostik nicht auf die lange Bank schieben}

$F^{\mathrm{r}}$ ragilitätsfrakturen werden oft nur unfallchirurgisch versorgt. Eine spezifische Osteoporosetherapie wird dagegen selten zeitnah initiiert. Die notwendige Abklärung wird dem Patienten überlassen, der sich damit häufig überfordert fühlt und die Dringlichkeit auch nicht erfasst. Daher seien Weiterbehandlungskonzepte für Patienten mit Osteoporose-assoziierten Frakturen wichtig, betonte Dr. Swantje Oberthür, Klinik für Unfallchirurgie, Orthopädie und Plastische Chirurgie, Universitätsklinik Göttingen. Der reine Verweis an osteologische Praxen reiche nicht aus.
In Göttingen konnte die Abklärungsrate auf eine Osteoporose als möglicher Ursache einer Fragilitätsfraktur durch einen im März 2016 eingerichteten Fracture Liaison Service (FLS) deutlich verbessert werden. Alle Patienten mit Osteoporose-verdächtiger Fraktur werden nun bereits in der Notaufnahme beziehungsweise in einer Spezialsprechstunde gescreent und über die Notwendigkeit einer Osteoporosediagnostik informiert. Interessierte werden in den FLS aufgenommen und erhalten bereits während des Krankenhausaufenthaltes eine Knochendichte-Messung
(DXA), Labor und Risikoevaluierung mittels des standardisierten Fragebogens des DVO (Dachverband Osteologie). Ambulante Patienten werden an niedergelassene osteologische Praxen vermittelt. Der Patient beziehungsweise sein Hausarzt erhalten nach der Auswertung einen FLS-Brief mit einer entsprechenden Therapieempfehlung. Wie eine inzwischen durchgeführte prospektive Untersuchung dokumentiert, hat sich Oberthür zufolge durch dieses Vorgehen die Rate der Abklärungen auf eine Osteoporose von 1,8\% bei alleiniger Information über die Notwendigkeit einer Osteoporosediagnostik plus Mitgeben von Adressen osteologischer Praxen auf immerhin $37 \%$ steigern lassen.

kat

\section{Periprothetische Infektion: Am TEP-Wechsel führt meist kein Weg vorbei}

$\mathrm{D}$ ie Chance auf eine erfolgreiche Therapie einer periprothetischen Infektion (PPI) hängt maßgeblich von der frühen Diagnose ab. Aber schon hier stellt sich das erste Problem, wie Prof. Carsten Perka, Ärztlicher Direktor des Centrums für Muskuloskeletale Chirurgie, Charité Universitätsmedizin, Berlin, erläuterte. Denn es gebe keine einheitliche Definition einer PPI. Wichtige Hinweise aus der Anamnese seien Wundheilungsstörungen in der Vergangenheit, verlängerte Antibiotikagabe und Revisionsoperationen mit verlängerter Wundsekretion. Als klinisch wichtigen Hinweis erachtet Perka die Schmerzbewertung durch den Patienten (Änderung von Schmerzcharakter und -intensität); der Parameter „Rötung“ sei wenig aussagekräftig, das Messen der Blutsenkungsgeschwindigkeit verzichtbar und in den meisten Kliniken weitgehend durch die Bestimmung des C-reaktiven Proteins (CRP) als Screeningverfahren verdrängt worden. CRP-Werte $>10 \mathrm{mg} / \mathrm{l}$ sechs Wochen nach der Operation können als Hinweis auf eine periprothetische Infektion gewertet werden.

Für die weitergehende Diagnostik ist eine schnellstmögliche Gelenkpunktion mit Aspiration inzwischen rechtlich gefordert und für die Bestimmung der
Leukozyten und Neutrophilen unverzichtbar. Das Aspirat sollte also bei ausreichender Menge nicht nur in die Mikrobiologie, sondern auch ins Labor geschickt werden. Für die mikrobiologische Untersuchung sollten möglichst mehr als fünf Proben für mindestens 10-14 Tage bebrütet werden. Eine gegebenenfalls bereits begonnene Antibiotikatherapie muss zuvor beendet werden, um die verursachenden Bakterien sicher detektieren und eine Resistenzbestimmung durchführen zu können. Perka zufolge ist die Sonikation bisher zwar kein Muss, aber insbesondere zur Detektion von Low-Grade-Infektionen empfehlenswert. Die Nachweisrate der Erreger könne erhöht werden, weil mittels Sonikation Bakterien aus dem Biofilm herausgelöst werden.

Die Untersuchung der Synovialflüssigkeit mit dem Alpha-Defensin-Bedsideoder dem Leukozytenesterase-Test können als Unterstützung hilfreich sein. Den D-Laktat-Test hält Perka noch nicht für praxistauglich. Die inzwischen preiswerte $(<200 €)$ Multiplex-Genomanalyse dauere derzeit noch zu lange, könne aber bald an Bedeutung gewinnen.

Der Gelenkerhalt ist laut Perka prinzipiell bis maximal 3-4 Wochen nach Beginn der PPI möglich, in den allermeis- ten Fällen aber keine adäquate Therapieoption. Bei Patienten mit mehreren Endoprothesen bestehe bei dieser Strategie die Gefahr einer Ausbreitung der Infektion auf weitere Kunstgelenke. Beim gelenkerhaltenden Vorgehen werden die beweglichen Endoprothesenteile ausgetauscht, ein chirurgisches Débridement mit Spülung und eine mindestens sechswöchige Antibiotikatherapie durchgeführt. Laut Perka liegt das Risiko für einen Fehlschlag aber bei $50: 50$. Im Fall von Problemkeimen sei es sogar noch höher, was vielen nicht klar sei.

Muss das Gelenk entfernt werden, sei der einzeitige Wechsel zwar generell die bessere Lösung für den Patienten und das Gesundheitssystem. Voraussetzung für den Erfolg dieser Strategie sei aber zum einen ein aggressives Débridement, was oft nicht durchführbar sei. Zum anderen werde ein adäquates, TotraumManagement' gefordert, das idealerweise durch eine Lappenplastik, im Klinikalltag aber meist durch Zement, Ketten oder Spacer erfolgt. Diese geben aber nur maximal zwei Wochen Antibiotika ab, sodass häufig erneut interveniert werden muss. Auch die Forderung nach einer Immunkompetenz des Patienten ist oft nicht erfüllt und vor allem nicht klar durch Laborparameter definiert. So bleibt Perka zufolge in vielen Fällen nur der zweizeitige Wechsel der Endoprothese. 